\title{
Map-Like Representations of an Abstract Conceptual Space in the Human Brain
}

\author{
Levan Bokeria*, Richard N. Henson and Robert M. Mok* \\ MRC Cognition and Brain Sciences Unit, University of Cambridge, Cambridge, United Kingdom
}

Keywords: conceptual space, concept learning, semantics, categorization, navigation, grid cells, entorhinal cortex

\section{INTRODUCTION}

Much of higher cognition involves abstracting away from sensory details and thinking conceptually. How do our brains learn and represent such abstract concepts? Recent work has proposed that neural representations in the medial temporal lobe (MTL), which are involved in spatial navigation, might also support learning of higher-level knowledge structures (Behrens et al., 2018; Bellmund et al., 2018). Under this view, a range of MTL neurons such as place cells, grid cells, and headdirection cells may support the ability to mentally "navigate" through conceptual spaces. This extends the original proposal by Tolman (1948) that people construct "cognitive maps" that support broad psychological functions, and offers the exciting potential of understanding the cognitive processes that underlie category learning, reinforcement learning, and spatial navigation under a single unified framework.

These ideas are supported by findings that neural representations in the MTL, as well as the medial prefrontal cortex (mPFC), are involved in "navigation" of simple two-dimensional spaces of visual stimuli (Constantinescu et al., 2016; Theves et al., 2019, 2020), social spaces (Tavares et al., 2015; Park et al., 2020), and odor spaces (Bao et al., 2019). A recent study in the Journal of Neuroscience (Viganò and Piazza, 2020) takes this research further by suggesting that the entorhinal cortex (EHC) and the mPFC are capable of mapping not only sensory spaces, but also abstract semantic spaces. In this opinion piece, we first describe the paradigm and results of Viganò and Piazza (2020), as well as the importance of their findings for the field. We then raise several methodological concerns and suggest changes to the paradigm to address these issues. Finally, we discuss potential future research directions including experimental and modeling approaches to tackle outstanding questions in the field.

\section{EXPERIMENTAL PARADIGM AND RESULTS}

Specialty section:

This article was submitted to Cognitive Neuroscience,

a section of the journal

Frontiers in Human Neuroscience

Received: 21 October 2020 Accepted: 04 January 2021 Published: 02 February 2021

Citation: Bokeria L, Henson RN and Mok RM (2021) Map-Like Representations of an Abstract Conceptual Space in the

Human Brain

Front. Hum. Neurosci. 15:620056. doi: 10.3389/fnhum.2021.620056

Viganò and Piazza (2020) employed a two-dimensional, multisensory space, in which each exemplar was characterized by the size of a visual image and the pitch of a concurrent auditory tone (Figures 1A,B). To assess how the brain organizes category knowledge in this space, they used functional magnetic resonance imaging (fMRI) to measure the distances and directions between the neural representations of exemplars, both before and after category learning. The experiment involved three phases: (1) a pre-learning fMRI task, (2) nine days of behavioral training on the category structure, and (3) a post-learning fMRI task. During pre-learning, participants performed a 1-back stimulus identity task on the exemplars and on verbal labels (the pseudowords KER, MOS, DUN, and GAL) that denoted the four later-to-be-learned categories (Figure 1B). During behavioral training, participants learned to map each exemplar to one of the four categories. The post-learning task was similar to the pre-learning task, except participants performed a 1back category identity task, responding whenever an exemplar was followed by its corresponding category label or vice versa. 
When searching for neural representations that related to the distances between categories, the authors utilized the fact that diagonal category pairs (such as DUN-MOS) differed in both dimensions and so were further apart than horizontal/vertical categories (such as KER-MOS) that differed in only one dimension (Figure 1C, left). They used fMRIadaptation to detect sensitivity to small vs. large distances, and representational similarity analysis (RSA) to assess similarity between neural representations across categories (Figure 1D, left). Both analyses revealed a significant cluster in the mPFC that reflected greater distance between diagonal categories than horizontal/vertical categories post-learning, but not prelearning (Figures 1C,D). An additional fMRI-adaptation analysis focusing on the EHC showed that the EHC also tracked category distances. Importantly, a control RSA based on distances between individual exemplars suggested that the MPFC was not sensitive to sensory distances between stimuli either pre- or post-learning, demonstrating its specificity to the more abstract category space.

The authors then used model-based RSA to test for directionbased grid-like neural codes for "movement" across the category space. A model was constructed to reflect the predicted similarities between all eight possible movement directions in the category space ("moving" from one trial to the next in the 1-back task). The similarities across directions were calculated assuming a 6-fold periodicity that would result from using a grid-like code (Figure 1E, left; see Bellmund et al., 2016; though note that only eight directions were sampled in this study). A whole-brain searchlight revealed a cluster in the right $\mathrm{EHC}$ consistent with this model (Figure 1E, right). This EHC signal was not sensitive to 4,5 , and 7 -fold periodicities, and was only present postlearning. Notably, the authors did not find a direction-modulated signal in the mPFC.

Together, these results suggest that the neural mechanisms that underlie spatial processing are recycled to represent not just sensory spaces but also more abstract category spaces. Specifically, the mPFC and the EHC represent category distances, and the EHC tracks the movement directions in that space, possibly using the same grid-like codes found in spatial navigation tasks.

\section{DISCUSSION}

Despite its innovative paradigm, aspects of the experimental design warrant further consideration. Specifically, the pre- vs. post-learning tasks differed in nature (1-back stimulus identity vs. 1-back category identity), which could have introduced confounds such as differences in task difficulty-i.e., greater brain activity due to higher cognitive demand in the category task. This is one reason why the authors did not report a direct prevs. post-learning whole-brain comparison, even though such a contrast revealed the same $\mathrm{mPFC}$ cluster as reported in the paper (personal communication, April 9, 2020). One way to address this issue would be to run the 1-back stimulus identity task postas well as pre-learning, attributing any changes in brain activity to the newly acquired category knowledge. This would allow a direct whole-brain comparison between pre- and post-learning stages, providing a better and more comprehensive test compared to the pre- post-learning comparison within the mPFC ROI identified by the analysis after learning (Figures 1C,D, right; also see Viganò and Piazza, 2020, Figures 2C,F). Indeed, the authors did run a post-learning 1-back stimulus identity task, but with fewer number of runs than pre-learning, undermining the power of the pre-post contrast (personal communication, April 9, 2020). Additionally, a post-learning 1-back stimulus identity task might not have activated the learned category knowledge since it is not required to perform the task. Thus, an alternative approach would be to introduce a within-participant "no-learning" control condition, where participants perform a pre-learning 1-back stimulus identity task and a post-learning 1back category identity task on a second, distinct set of stimuli and category labels. Crucially, participants would not learn categoryto-exemplar mappings in between the tasks (such that in the post-learning task, participants would need to guess the category to which exemplars belong). Then, one could examine distance and direction sensitivity that was unique to the "learned" space.

Another issue concerns the possibility that the directionbased, grid-like representations of concept space may be partially driven by more concept-level overlap in one direction than others. With four categories arranged in a square format, only a subset of directions across concepts could be sampled (0$315^{\circ}$ in $45^{\circ}$ steps $)$, thereby omitting several key directions $\left(60^{\circ}\right.$ and $120^{\circ}$ ) when testing for a 6 -fold periodic signal (Figure 1E, left). Therefore, the significant correlation with the grid-model could have been mainly driven by the higher pattern similarity (lower dissimilarity) in the $180^{\circ}$ direction. The issue is that the conditions that are compared in the $180^{\circ}$ direction contained stimuli that shared two concept labels (e.g., DUN $\rightarrow$ GAL then $\mathrm{GAL} \rightarrow \mathrm{DUN}$ ) whereas the other direction conditions only shared one concept label (e.g., one $45^{\circ}$ movement is from DUN $\rightarrow$ KER then $\mathrm{KER} \rightarrow \mathrm{GAL}$, sharing $\mathrm{KER}$ ). If the brain region carries information about the concept, the $180^{\circ}$ direction would show higher pattern similarity than the other directions whether or not there is an underlying grid-like signal. One hint of evidence supporting a grid-like representation the right EHC is that the pattern dissimilarities appear to increase from "zero" to "small" to "large" changes in the predicted grid-model RDM (Figure 1E, right, bar-plot inset). Although the numerical trend matches the 6-fold prediction, it is unclear whether the pattern dissimilarities for "small" and "large" are statistically distinguishable, and the main result could still be driven by the small dissimilarities in the "zero" condition (i.e., $180^{\circ}$ ). Future work could use a larger set of exemplars and concept labels to test a wider range of movement directions and hence provide further evidence for the emergence of a grid-like representation in concept space after learning.

It is notable that Viganò and Piazza (2020) did not find distance-based representations in the hippocampus, for either category or sensory spaces. Structures in the MTL have been implicated in a wide range of cognitive functions from spatial navigation to memory to concept formation. We provide a brief overview of these functions and speculate on the possible reasons for the absence of hippocampal involvement in the current study. First, the hippocampus is a region strongly implicated in spatial navigation, where place cells are thought 
A.

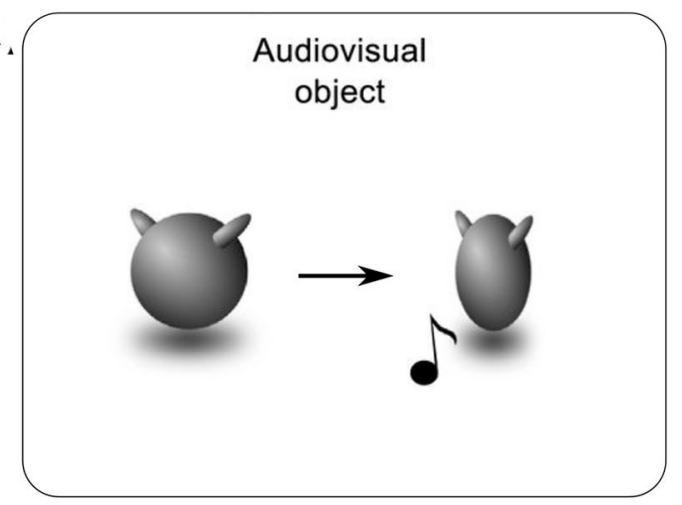

c

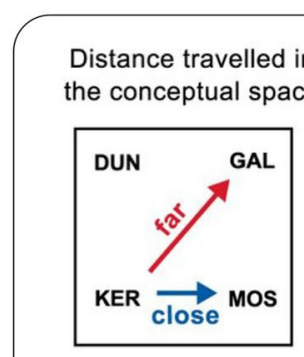

Distance travelled in

Group-level whole-brain adaptation

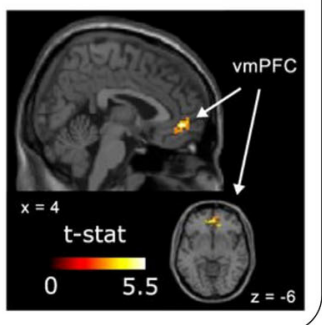

$\mathrm{E}$

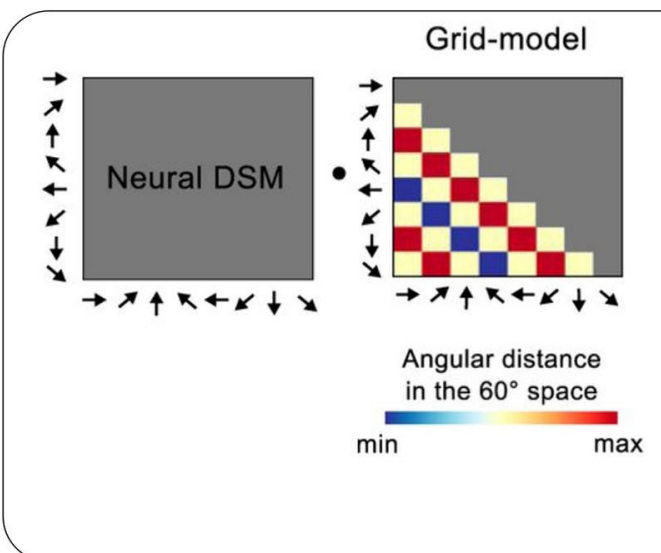

B

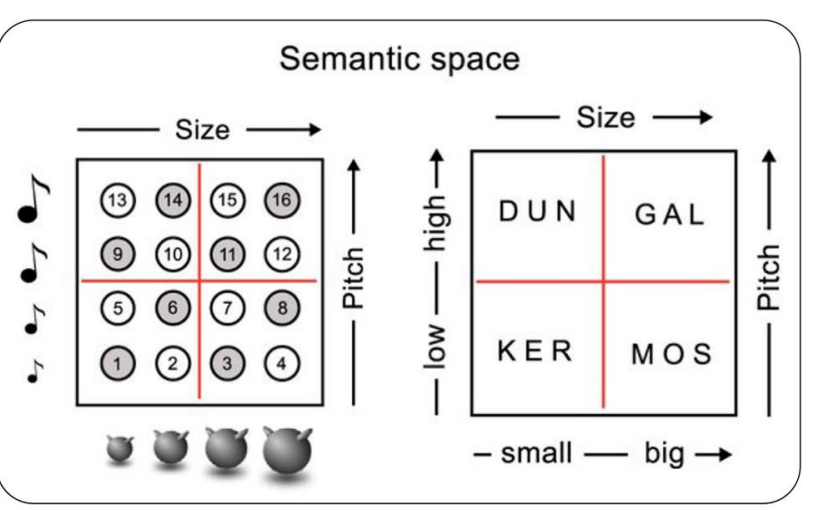

D

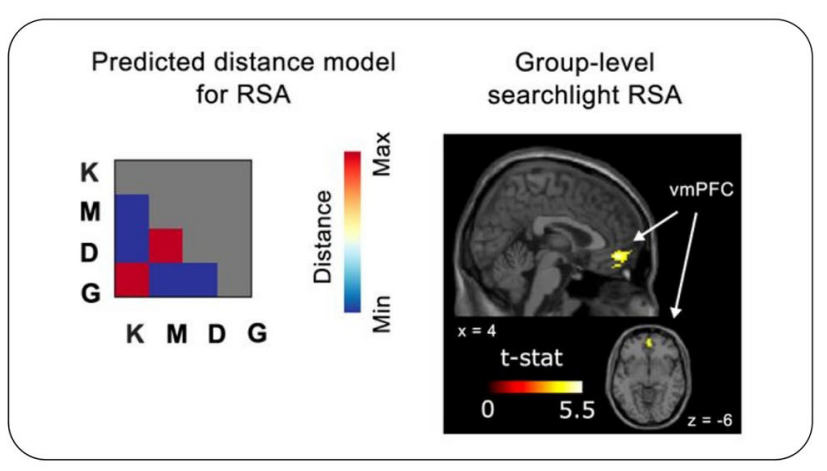

Searchlight results

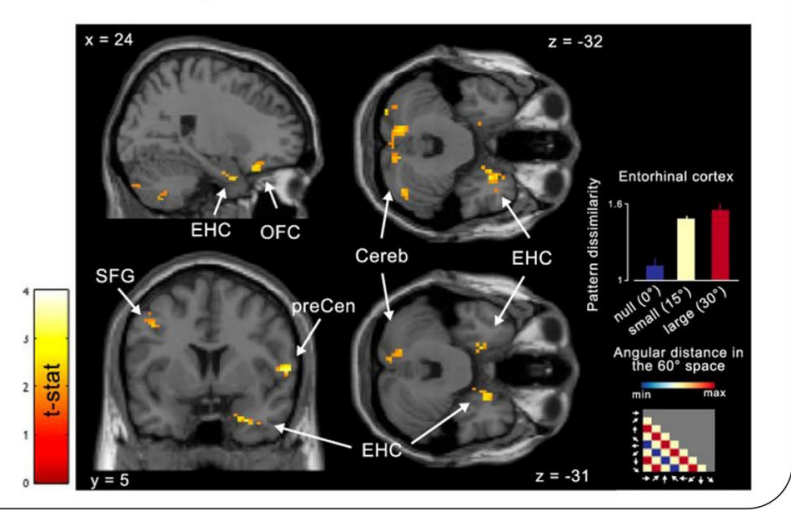

FIGURE 1 | Task and results summary. (A) An example audiovisual object used in the experiment. Each object was of a particular size and made a sound of a specific pitch as it visually compressed on the screen. (B) Left: Illustration of the 16 objects in a 2D audiovisual sensory space defined by size and pitch. Right: Objects were assigned one of four category labels (meaningless pseudowords), which defined the abstract semantic/category space on top of the sensory space. Objects marked with white and gray circles were both used in the behavioral sessions; only the ones marked in gray were used in the fMRI sessions. (C,D) Brain representations coding semantic distance. (C) Left: Illustration of greater distances in semantic space between diagonal categories (here KER-GAL) than non-diagonal distances (here KER-MOS). Right: Whole-brain fMRI-adaptation analysis performed on post-learning data revealed a significant cluster in the mPFC. (D) Left: Representational similarity analysis (RSA) using a model based on distances between categories. Right: Whole-brain RSA searchlight revealed a significant cluster in the mPFC. (E) Grid-like representations of semantic space. Left: Assuming a grid-like representation in semantic space, a specific pattern of activation across different movement directions should be expected (where movement refers to transitions between stimuli on successive trials). A grid-model dissimilarity matrix represents the predicted pairwise dissimilarities between voxel activity patterns evoked by the different directions as a function of their angular distance in $60^{\circ}$ periodic space. This grid-model is then correlated with the voxel activity patterns evoked by the different movement directions to reveal the presence of a grid-like response. Right: whole-brain searchlight results using the model-based grid-RSA. Maps are thresholded at $p<0.05$ uncorrected for visualization. Bar plot inset shows the average dissimilarity between activity patterns evoked by different directions in the entorhinal cortex, varying as a function of angular distance modulo 60. The figure is adapted from Viganò and Piazza (2020), Journal of Neuroscience. 
to track an agent's physical location (O'Keefe and Nadel, 1978). Relatedly, human neuroimaging studies have implicated the hippocampus in tracking distances in virtual spaces (Deuker et al., 2016), conceptual spaces (Tavares et al., 2015; Theves et al., 2019, 2020) and graph-like relational knowledge structures (Garvert et al., 2017). The MTL has long been acknowledged for its role in learning and memory (Nadel and Moscovitch, 1997). MTL structures such as the hippocampus, EHC, parahippocampal cortex, and perirhinal cortex are involved in the integration of multiple sources of information such as object identity and spatio-temporal context into a coherent episodic event representation that can be consolidated into memory (Eichenbaum and Cohen, 2008; Knierim et al., 2014). Interestingly, the role of the MTL in semantic memory has been debated (Squire and Zola-Morgan, 1991; Mishkin et al., 1998; Duff et al., 2020) with some researchers arguing for the anterior temporal lobe (ATL) as the crucial region for representing semantic knowledge, supported by semantic deficits in semantic dementia (Ralph et al., 2016). Recent work also showed the involvement of MTL structures such as the hippocampus in concept learning and categorization (Davis et al., 2012; Mack et al., 2016; Bowman and Zeithamova, 2018), with theoretical proposals arguing for an intrinsic link between episodic memory and concept formation (Mack et al., 2018). One reason why the hippocampus was not identified in the experiment by Viganò and Piazza (2020) may be that the new category knowledge had already become consolidated into cortex over the nine days of training, and therefore was no longer hippocampally dependent (Squire et al., 2015). Consistent with this, studies that showed hippocampal involvement in concept learning tested participants relatively early in the learning phase (Davis et al., 2012; Mack et al., 2016). On the other hand, involvement of the EHC in representing conceptual information after long periods of learning as show in Viganò and Piazza's study can inform the debate about the possible role of the MTL structures in organizing semantic knowledge. Future studies could clarify the differential contributions of these MTL structures by tracking neural representations at multiple stages of learning, before and after consolidation.

Another way to uncover the neural representations underlying abstract concepts is to develop computational models that also learn categories (Nosofsky, 1986; Kruschke, 1992; Nosofsky et al., 1994; Ashby et al., 1998; Smith and Minda, 1998; Love et al., 2004; Sanborn et al., 2010), and search the brain for representations that match those learned by the models. This approach has focused on the hippocampus (Mack et al., 2016; Bowman and Zeithamova, 2018) though a recent model based on clustering theories of concept learning was able to capture both hippocampal representations in category learning tasks and place and grid cell-like representations in navigation contexts (Mok and Love, 2019). Applied to a concept structure similar to the one in the current study, the model would learn the centers of the concept distributions (representing the category prototypes), and predict stronger activity for category prototypes than nonprototypes, and more similar representations for movements between category prototypes compared to movements toward non-prototype exemplars. This prediction could not be tested in the current study because the exemplars were equally distant from their category prototype. By expanding the sensory space to include more exemplars and testing more movement directions, future studies may be able to distinguish this model from others, by comparing the similarity spaces within brain regions with similarity spaces implied by different computational models (e.g., spatial, clustering, exemplar, Bayesian models).

While brain-imaging studies provide growing evidence for general-purpose neural mechanisms across spatial and conceptual domains, an older line of behavioral work challenges these ideas by showing that people violate axiomatic assumptions in geometric theories of conceptual organization (Tversky and Gati, 1982). If conceptual spaces are represented geometrically (Gärdenfors, 2000; Bellmund et al., 2018), then they must obey several geometric properties, such as the "triangle inequality." This states that a direct path between two points cannot be larger than an indirect one going through a third point. Using two-dimensional stimuli similar to ones used in neuroimaging studies (e.g., Constantinescu et al., 2016), Tversky and Gati (1982) showed that people's judgments of the dissimilarity between pairs of exemplars imply internal conceptual spaces that violate the triangle inequality. While augmented geometric models have been developed to address this problem (Krumhansl, 1978; Nosofsky, 1991), some theoretical issues remain (Goldstone and Son, 2012). Therefore, proposals that suggest that spatial representations underlie human concept learning need to address this challenge to resolve the tension between neural data and human behavioral data.

In summary, Viganò and Piazza (2020) provide an important contribution to the growing evidence for shared neural mechanisms underlying spatial navigation and abstract knowledge. Future paradigms should further disentangle the contributions of the hippocampus, the EHC, and the $\mathrm{mPFC}$ to the representation of category vs. sensory spaces, assess learning and consolidation-dependent changes in neural representations, and compare computational models in their ability to match neural and behavioral data. Together, these developments will clarify the extent to which we can generalize mechanistic insights from spatial navigation to the organization of higher-order knowledge structures.

\section{AUTHOR CONTRIBUTIONS}

$\mathrm{LB}, \mathrm{RH}$, and RM contributed to the conceptual argument developed in the opinion piece. LB and RM wrote the first draft of the manuscript. All authors contributed to manuscript revision and read and approved the submitted version.

\section{FUNDING}

This work was supported by the Medical Research Council UK (SUAG/046 G101400 for RH and LB, SUAG/045 G101400 for $\mathrm{RM}$ ), by the Gates Cambridge Trust (to LB), and the Leverhulme Trust and the Isaac Newton Trust (SUAI/053 G100773, SUAI/056 G105620, ECF-2019-110 to RM). 


\section{ACKNOWLEDGMENTS}

We would like to thank Jascha Achterberg for his feedback and thoughts on the paper drafts. We would also like to acknowledge Simone Viganò for an extensive and thorough

\section{REFERENCES}

Ashby, F. G., Alfonso-Reese, L. A., Turken, A. U., and Waldron, E. M. (1998). A neuropsychological theory of multiple systems in category learning. Psychol. Rev. 105, 442-481. doi: 10.1037/0033-295X.105.3.442

Bao, X., Gjorgieva, E., Shanahan, L. K., Howard, J. D., Kahnt, T., and Gottfried, J. A. (2019). Grid-like neural representations support olfactory navigation of a two-dimensional odor space. Neuron 102, 1066-1075.e5. doi: 10.1016/j.neuron.2019.03.034

Behrens, T. E. J., Muller, T. H., Whittington, J. C. R., Mark, S., Baram, A. B., Stachenfeld, K. L., et al. (2018). What is a cognitive map? Organizing knowledge for flexible behavior. Neuron 100, 490-509. doi: 10.1016/j.neuron.2018.10.002

Bellmund, J. L. S., Deuker, L., Schröder, T. N., and Doeller, C. F. (2016). Grid-cell representations in mental simulation. Elife 5:e17089. doi: $10.7554 /$ eLife. 17089.028

Bellmund, J. L. S., Gärdenfors, P., Moser, E. I., and Doeller, C. F. (2018). Navigating cognition: spatial codes for human thinking. Science 362:eaat6766. doi: 10.1126/science.aat6766

Bokeria, L., Henson, R., and Mok, R. M. (2020). Map-like representations of an abstract conceptual space in the human brain. PsyArXiv. doi: 10.31234/osf.io/2yb7a

Bowman, C. R., and Zeithamova, D. (2018). Abstract memory representations in the ventromedial prefrontal cortex and hippocampus support concept generalization. J. Neurosci. 38, 2605-2614. doi: 10.1523/JNEUROSCI.2811-17.2018

Constantinescu, A. O., O'Reilly, J. X., and Behrens, T. E. J. (2016). Organizing conceptual knowledge in humans with a gridlike code. Science 352, 1464-1468. doi: 10.1126/science.aaf0941

Davis, T., Love, B. C., and Preston, A. R. (2012). Learning the exception to the rule: model-based fMRI reveals specialized representations for surprising category members. Cerebral Cortex 22, 260-273. doi: 10.1093/cercor/bhr036

Deuker, L., Bellmund, J. L., Navarro Schröder, T., and Doeller, C. F. (2016). An event map of memory space in the hippocampus. ELife 5:e16534. doi: $10.7554 /$ eLife. 16534.018

Duff, M. C., Covington, N. V., Hilverman, C., and Cohen, N. J. (2020). Semantic memory and the hippocampus: revisiting, reaffirming, and extending the reach of their critical relationship. Front. Hum. Neurosci. 13:471. doi: 10.3389/fnhum.2019.00471

Eichenbaum, H., and Cohen, N. J. (2008). From Conditioning to Conscious Recollection: Memory Systems of the Brain. Oxford: Oxford University Press.

Gärdenfors, P. (2000). Conceptual Spaces: The Geometry of Thought. Cambridge, MA: The MIT Press.

Garvert, M. M., Dolan, R. J., and Behrens, T. E. J. (2017). A map of abstract relational knowledge in the human hippocampal-entorhinal cortex. Elife 6:e17086. doi: 10.7554/eLife.17086.021

Goldstone, R. L., and Son, J. Y. (2012). "Similarity",in The Oxford Handbook of Thinking and Reasoning, eds K. J. Holyoak and R. G. Morrison (Oxford: Oxford University Press) 155-176. doi: 10.1093/oxfordhb/9780199734689.013.0010

Knierim, J. J., Neunuebel, J. P., and Deshmukh, S. S. (2014). Functional correlates of the lateral and medial entorhinal cortex: Objects, path integration and local - global reference frames. Philos. Trans. R. Soc. B 369:20130369. doi: $10.1098 /$ rstb.2013.0369

Krumhansl, C. L. (1978). Concerning the applicability of geometric models to similarity data: the interrelationship between similarity and spatial density. Psychol. Rev. 85, 445-463. doi: 10.1037/0033-295X.85.5.445

Kruschke, J. K. (1992). ALCOVE: an exemplar-based connectionist model of category learning. Psychol. Rev. 99, 22-44. doi: 10.1037/0033-295X.99.1.22 personal communication on the experimental paradigm, results and interpretations of the reviewed Viganò and Piazza (2020) paper, and for agreeing to have this communication referenced here. The contents of this article have previously appeared as a preprint which can be found at Bokeria et al. (2020).
Love, B. C., Medin, D. L., and Gureckis, T. M. (2004). SUSTAIN: a network model of category learning. Psychol. Rev. 111, 309-332. doi: 10.1037/0033-295X.111.2.309

Mack, M. L., Love, B. C., and Preston, A. R. (2016). Dynamic updating of hippocampal object representations reflects new conceptual knowledge. Proc. Natl. Acad. Sci. U.S.A. 113, 13203-13208. doi: 10.1073/pnas.1614048113

Mack, M. L., Love, B. C., and Preston, A. R. (2018). Building concepts one episode at a time: the hippocampus and concept formation. Neurosci. Lett. 680, 31-38. doi: 10.1016/j.neulet.2017.07.061

Mishkin, M., Vargha-Khadem, F., and Gadian, D. G. (1998). Amnesia and the organization of the hippocampal system. Hippocampus 8, 212-216. doi: 10.1002/(SICI)1098-1063(1998)8:3<212::AID-HIPO4>3.0.CO;2-L

Mok, R. M., and Love, B. C. (2019). A non-spatial account of place and grid cells based on clustering models of concept learning. Nat. Commun. 10:5685. doi: 10.1038/s41467-019-13760-8

Nadel, L., and Moscovitch, M. (1997). Memory consolidation, retrograde amnesia and the hippocampal complex. Curr. Opin. Neurobiol. 7, 217-227. doi: 10.1016/S0959-4388(97)80010-4

Nosofsky, R. M. (1986). Attention, similarity, and the identification-categorization relationship. J. Exp. Psychol. 115, 39-57. doi: 10.1037/0096-3445.115.1.39

Nosofsky, R. M. (1991). Stimulus bias, asymmetric similarity, and classification. Cogn. Psychol. 23, 94-140. doi: 10.1016/0010-0285(91)90004-8

Nosofsky, R. M., Palmeri, T. J., and McKinley, S. C. (1994). Rule-plusexception model of classification learning. Psychol. Rev. 101, 53-79. doi: 10.1037/0033-295X.101.1.53

O’Keefe, J., and Nadel, L. (1978). Hippocampus as a Cognitive Map. Oxford: Oxford University Press.

Park, S. A., Miller, D. S., Nili, H., Ranganath, C., and Boorman, E. D. (2020). Map making: constructing, combining, and inferring on abstract cognitive maps. Neuron 107, 1226-1238.e8. doi: 10.1016/j.neuron.2020.06.030

Ralph, M. A. L., Jefferies, E., Patterson, K., and Rogers, T. T. (2016). The neural and computational bases of semantic cognition. Nat. Rev. Neurosci. 18, 42-55. doi: $10.1038 /$ nrn.2016.150

Sanborn, A. N., Griffiths, T. L., and Navarro, D. J. (2010). Rational approximations to rational models: alternative algorithms for category learning. Psychol. Rev. 117, 1144-1167. doi: 10.1037/a0020511

Smith, J. D., and Minda, J. P. (1998). Prototypes in the mist: the early epochs of category learning. J. Exp. Psychol. 24, 1411-1436. doi: 10.1037/0278-7393.24.6.1411

Squire, L., and Zola-Morgan, S. (1991). The medial temporal lobe memory system. Science 253, 1380-1386. doi: 10.1126/science.1896849

Squire, L. R., Genzel, L., Wixted, J. T., and Morris, R. G. (2015). Memory consolidation. Cold Spring Harbor Perspect. Biol. 7:a021766. doi: $10.1101 /$ cshperspect.a021766

Tavares, R. M., Mendelsohn, A., Grossman, Y., Williams, C. H., Shapiro, M., Trope, Y., et al. (2015). A map for social navigation in the human brain. Neuron 87 , 231-243. doi: 10.1016/j.neuron.2015.06.011

Theves, S., Fernandez, G., and Doeller, C. F. (2019). The hippocampus encodes distances in multidimensional feature space. Curr. Biol. 29, 1226-1231.e3. doi: 10.1016/j.cub.2019.02.035

Theves, S., Fernández, G., and Doeller, C. F. (2020). The hippocampus maps concept space, not feature space. J. Neurosci. 40, 7318-7325. doi: 10.1523/JNEUROSCI.0494-20.2020

Tolman, E. C. (1948). Cognitive maps in rats and men. Psychol. Rev. 55, 189-208. doi: $10.1037 /$ h0061626

Tversky, A., and Gati, I. (1982). Similarity, separability, and the triangle inequality. Psychol. Rev. 89, 123-154. doi: 10.1037/0033-295X.89.2.123 
Viganò, S., and Piazza, M. (2020). Distance and direction codes underlie navigation of a novel semantic space in the human brain. J. Neurosci. 40, 2727-2736. doi: 10.1523/JNEUROSCI.1849-19.2020

Conflict of Interest: The authors declare that the research was conducted in the absence of any commercial or financial relationships that could be construed as a potential conflict of interest.
Copyright (C) 2021 Bokeria, Henson and Mok. This is an open-access article distributed under the terms of the Creative Commons Attribution License (CC BY). The use, distribution or reproduction in other forums is permitted, provided the original author(s) and the copyright owner(s) are credited and that the original publication in this journal is cited, in accordance with accepted academic practice. No use, distribution or reproduction is permitted which does not comply with these terms. 